\title{
Design and Implementation of FPGA-Based Digital Frequency Meter
}

\author{
HUANG rui ${ }^{1, \mathrm{a}}$ \\ ${ }^{1}$ Chongqing college of electronic engineering, Chongqing, 401331,China) \\ a2231066194@qq.com
}

Keywords: FPGA, Digital Frequency Meter, Design and Implementation

\begin{abstract}
Development of EDA technology has changed the traditional electronic design methods. Widely used in large-scale FPGA and other programmable logic devices, electronic design and software programming becomes as easy and quick. Advances in electronic design technology, but also changed the traditional frequency meter design. It exists conventional frequency measurement method using an analog circuit frequency characteristic measurement frequency, but also the use of a digital pulse counting frequency measurement method. With the development of digital circuit technology to pulse count based on the law derived from a variety of improved digital frequency measurement method, measurement accuracy, fast response measurement and other aspects have been greatly improved. This paper analyzes the direct frequency measurement method and measurement principle cycle frequency measurement method, which are explained in frequency measurement method of producing \pm 1 count error reasons.
\end{abstract}

\section{Introduction}

Frequency meter design technology with the development of electronic circuit technology and gradually move forward, the early frequency meter design using discrete components, long design cycles, poor stability, and the finished product volume, high power consumption. Development of digital electronic technology and integrated circuits, so that the digital frequency meter wide applications, digital frequency meter circuit and microcontroller unit can be used to design and implementation. Compared to a discrete piece of frequency meter, a digital frequency meter to improve the stability, reducing the volume, but there is still a digital frequency meter circuit complex, long design cycles and other shortcomings, digital frequency meter measuring range is limited, to measure signals of different frequencies are designed to be a part of the circuit, poor flexibility. 20th century, with the development of microelectronics technology advances and computer technology, both in the mutual promotion to FPGA family, represented by application of programmable logic devices becoming more common. Programmable logic device programmed by the universal integrated circuit integrated onto a single silicon chip the size of a small, exponentially reducing the size of the circuit, and because traces short, reduce interference and increase system reliability. Because such devices can be reconstructed through software programming and its structure and working methods of the hardware, such as hardware design software design as convenient, it brings a great deal of flexibility in the design of digital circuit system. With the integration of programmable logic devices have been expanding, continuously improve and enhance their own computer-aided design technology capabilities, EDA in modern electronic system design field was born.

\section{Study of Frequency Measurement Technique}

Currently, many methods for measuring the frequency accuracy of the measurement depends on the frequency characteristics of the frequency range of the measurement and the measured object. The measurement accuracy can be achieved, as the clock frequency depends not only on the accuracy of the criteria used, but also on measuring apparatus and methods used. Next, several types of commonly is used frequency measurement methods briefly. Direct Reading frequency measurement using the frequency characteristics of the circuit is the frequency interpretation. Direct Reading measured frequency of the bridge frequency measurement method, resonance frequency measurement method, frequency - voltage conversion frequency measurement method. 
Comparison of frequency measurement method is to use standard frequency fc and compare the measured frequency fx, when the standard frequency is adjusted to the measured frequency equal means zero meter (zero indicator) it means zero, and the value that is measured frequency standard frequency value. Comparison of measured frequency can be divided into the beat frequency measured and difference frequencies measured frequency models. The former is to be measured frequency signal and standard frequency signal superimposed on the linear element to generate a beat frequency. The latter is to be measured frequency signal and standard frequency signal in the non-linear mixing element. Currently beat frequency absolute error measurement frequency of about a few tenths of hertz, the frequency of the difference frequency measurement error can be orders of magnitude better than 10-5, the minimum detectable signal level of $0.1 \mu \mathrm{V} \sim 1 \mu \mathrm{V}$. Beat frequency and differential frequency method in routine situations rarely used.

Measurement frequency counting method is commonly used in digital frequency meter, the basic frequency measurement method. Count is the number per unit time of the periodic signal is counted. The accuracy of counting frequency measurement depends on the accuracy and error counts the reference time. The design uses a counting frequency measurements, the following notation for frequency measurement and frequency measurement principle based on several methods of counting method for detailed analysis.

\section{FPGA Structure and Working Principle}

FPGA is a field programmable logic array (Field Programmable Gate Array) abbreviation. It CPLD (Complex Programmable Logic Device, complex programmable logic devices), is currently used for a class of large-scale programmable logic devices.

For combinational logic circuit, the current output is always a function of the current input, and when the fixed circuit configuration, the same amount of input, output does not change. The combinational logic circuits are shown in Fig.1.

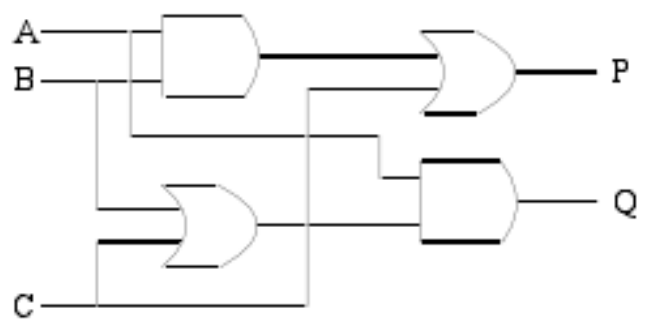

Fig.1 Combinational logic circuit examples

Truth table determines the logical relationship between input and output. The truth table and random access memory link, we will see if the input $\mathrm{A}, \mathrm{B}, \mathrm{C}$ as random access memory address input A2, A1, A0, the output P, Q can be seen as corresponding to the address memory the data stored in the storage unit D1, D0. . The memory cells each store different data, data (output) Address (input) and the storage unit may be constituted of a combination of different logic. This basic principle is implemented with random access memory (RAM) programmable combinational logic. FPGA programmable logic forming method is based on this principle programmable lookup tables (LookUp Table, LUT) structure. LUT is programmable logic constitutes the smallest unit. Most of the structure is formed using FPGA-based SRAM (static random access memory) look-up table logic, SRAM is used to form the logic function generator. Currently FPGA Multi-use 4-input LUT, so every LUT can be viewed as a 4-bit address lines $16 \times 1$ of RAM. All possible outcomes when the user development environment by the EDA schematic or HDL language to describe a logic circuit, the development of EDA software will automatically calculate the logic circuit, and the results pre-write RAM, so that each input signal logic operation is equivalent to input an address look-up table to find the address of the corresponding content, then the output can. 


\section{FPGA-Based Frequency Meter Design}

Frequency measurement and display the entire frequency meter control module is the core module for the fully digital circuit, using FPGA devices using VHDL programming to achieve. Frequency measurement part of multi-period synchronous frequency measurement method which achieve equal precision frequency measurement, measurement accuracy only and the reference frequency signal frequency, accuracy and relevant gate opening time, and frequency of the signal is independent, enabling a wide range of equal precision frequency measurement. The signal frequency measurement portion from the signal shaping module, as all pulse counting frequency measurement can only receive pulse signal, the signal must be shaped to form a pulse signal and the signal with the frequency. Reference frequency measurement part (clock) signal generated by the "reference frequency (clock) signal modules." Gate signal generator preset gate signal, because it is programmed, long can be programmed to modify all open gate signal. Operator by the formula $\mathrm{fx}=$ $(\mathrm{Nx} / \mathrm{Nc}) \cdot \mathrm{fc}$ calculate the frequency value of the measured signal, the operator of multiplication and division operations include two operations. The display control section includes a BCD code converter, decoder latch two sub-modules. Frequency operator is obtained in binary form, to be displayed in decimal form and it must be BCD code converter, in the form of BCD code to decimal. Frequency meter display portion using 7-segment, so the decoder, the latch complete display decoder BCD code, and also has stored decoding function.

The module generates a reference frequency signal frequency measurement using the standard clock pulse signal, also known as the clock signal module. The module uses a crystal oscillator and the corresponding hardware circuit to generate the standard clock signal, in addition to providing a reference clock signal frequency signal frequency measurement section 1 of the counter, but also provides the timing clock signal to the gate signal generator.

The form of the signal is varied, based on the pulse count after the frequency measurement can only accept pulse frequency signal, the signal must be shaped to form the measured signal with the pulse signal frequency to frequency values measuring. Frequency response characteristic of the signal shaping module also determines the frequency measurement frequency range of the upper frequency.

To simplify the design, frequency meter to display only the integer portion of the measured signal frequency value. Display module uses six seven-segment LED display. Because the seven-segment decoder, the latch circuit has the frequency measurement display module design is completed, the six digital tube directly to the FPGA pins to the corresponding output, but also consider the issue of digital drive tube.

Power modules provide the required standard for the entire voltage frequency meter modules. After the power module 220V AC power input, transformer rectifier, regulator device through the output DC voltage required by the system stable.

\section{Frequency Meter Design and Implementation of the Functional Modules}

Frequency measurement and display control module design using top-down design approach. First, the module is according to the functions are divided into several sub-modules, and then were using VHDL, and finally through the top-level design file to each sub-module assembled together to complete frequency measurement and display control functions.

A reference frequency signal is measured based on the frequency, and in the design of the reference frequency signal is also used as a timing clock signal multiplier, divider, BCD code converters, etc. Cyclone series FPGA devices contained in the present design uses high-performance embedded analog phase-locked loop (DPLL performance is far superior), the phase-locked loop (PLL) can be synchronized with the clock signal input, and the input clock as PLL reference implementation, and the input clock frequency multiplier or do 1 to 32 division. Compared with the clock directly from the external, internal clock this chip can reduce the clock delay and clock distortion, reduce chip interference, but also can improve the set-up time and hold 
time clock.

Although the measured signal is a periodic signal, its form may be varied. But the frequency measurement signal module can only be measured in the form of pulses, so before the measurement to the measured signal shaping, the different forms of signals organized into periodic pulse signal.

Frequency measurement system consists of different chips, each chip required supply voltage have different requirements, so the requirements of the power supply module to provide multiple voltage value. FPGA devices are used in this design Altera's EP1C3T144C8, core voltage of the device is $1.5 \mathrm{~V}$, I / O voltage of $3.3 \mathrm{~V}$, FPGA need to provide $+1.5 \mathrm{~V}$ and $+3.3 \mathrm{~V}$ dual voltage; inverting oscillator circuit 74LS04 devices require + 5V voltage; LM361 the standard voltage Vcc is $+5 \mathrm{~V}, \mathrm{~V}+$ and $\mathrm{V}$ - voltage range is wide, $\mathrm{V}+$ requires $5 \mathrm{~V} \sim 15 \mathrm{~V}, \mathrm{~V}$ - between $-6 \mathrm{~V} \sim-15 \mathrm{~V}$.

The design of frequency values are measured by the six seven-segment LED display. Since the latch function display decoder and decoding of digital values in the FPGA design has been completed, so the FPGA output can directly access input digital tube display frequency value. However, due to limited FPGA drive capability, it said only a theoretical point of view, the actual application, but also to access the digital front tube drive circuit, here we use ULN2003 as digital tube driver.

\section{Conclusion}

Frequency meter is one of the commonly used electronic measuring equipment. With the development of electronic technology, design methodology frequency meter are gradually moving forward. Discrete pieces from the early to the integrated circuit, LSI, this process must have the appropriate design features components to complete. When the development of electronic design EDA technology, the emergence of large-scale, ultra-large-scale programmable logic device, the electronic circuit includes a frequency meter design becomes more simple and convenient, and without changing the hardware structure on the basis and may be changed according to design requirements design, different functions and even complete systems with the same hardware circuit design. Based on the analysis and comparison of several commonly used based on the frequency measurement method, synchronous frequency measurement method according to the design need to use a multi-cycle frequency meter design. As a result of EDA design technology, most of the design work is done on the computer and logic simulation is completed by the middle of the computer.

\section{References}

[1] W. Zhou. Systematic Reseach on High-Accuracy Frequency Measurements and Control. Shizuoka University.Doctor Dissertation, 2000:15-24,90-96

[2] M.A.A .Sour, H.I.Saleh. An FPGA Inplem Entation Guide for Some Different Types Ofserial-Parallel Multiplier Structures. Microelectronics Fournal, 2000, 31(3):161-168

[3] W.Zhou. Some New Method for Precision Time Interval Measurement. Proceedings Of The1977 Ieee International Frequency Control Symposium, 1997,418-421

[4] R.A.James, F. Gallgray. Vhdl Design Expression and Synthesis of Vhdl. Cambridge: Harvard University Press, 2001

[5] C.Altera. Configuration Devices For Sram-Based Lut Devices. 2002.

[6] F.W.John. Digital Design Principles And Practices,2001.

[7] M.M.Mano. Digital Design, 3rd Ed. Prentice Hall Usa,2002. 